\title{
Conhecimento materno sobre o desenvolvimento infantil no contexto rural
}

\author{
Maternal knowledge about child development in the rural context \\ Conocimiento materno sobre el desarrollo infantil en el contexto rural
}

Jéssica Rodrigues de Sousa ${ }^{1}$, Emanuele Rocha da Silva ${ }^{1}$, Katiane da Costa Cunha ${ }^{2}$, Aurimery Gomes Chermont $^{1}$, Victor Kenji Medeiros Shiramizu ${ }^{3}$, Ivete Furtado Ribeiro Caldas ${ }^{2 *}$.

\section{RESUMO}

Objetivo: Investigar os fatores ambientais e sociodemográficos relacionados ao conhecimento materno sobre o desenvolvimento infantil, no contexto rural. Métodos: Estudo transversal, envolvendo mães de filhos de 0 a 3 anos de idade em município do Pará. Utilizou-se ficha de identificação, caracterização ambiental e sociodemográfica e o Knowledge of Infant Development Inventory (KIDI), aplicados por entrevista. Para análise estatística, utilizou-se modelos lineares generalizados; para comparações múltiplas o teste Sidak e o Qui-Quadrado para características amostrais. O nível de significância foi de 0,01\%. O estudo foi aprovado por Comitê de Ética em Pesquisa. Resultados: Para as 25 mães incluídas no estudo, o saneamento básico foi o único com significância estatística. Para as pontuações percentuais e desvio padrão, houve acertos sobre as perguntas com $0.90( \pm 0.07)$ na tentativa, $0.57( \pm 0.07)$ na precisão e 0.51 $( \pm 0.07)$ na pontuação total. Ainda, $0.34( \pm 0.08)$ das mães subestimaram o desenvolvimento de seus filhos e $0.14( \pm 0.06)$ superestimaram. Conclusão: $O$ conhecimento das mães da zona rural sobre 0 desenvolvimento infantil está relacionado a fatores sociodemográficos, sendo saneamento básico, dentre as variáveis ambientais, o único preditor da precisão e do total de acertos no instrumento, indicando predizer maior conhecimento materno quando presente.

Palavras-chave: Conhecimentos, Atitudes e prática em saúde, Desenvolvimento Infantil, Características culturais, área rural.

\begin{abstract}
Objective: To investigate the environmental and sociodemographic factors related to maternal knowledge about child development in the rural context. Methods: Cross-sectional study involving mothers of children between 0 and 3 years old, in a Pará state municipality. Environmental and sociodemographic characterization sheet and the Knowledge of Infant Development Inventory (KIDI) were used as an interview. Generalized linear models were used for statistical analysis; for multiple comparisons, the Sidak test, and the characteristics of the sample were compared through Chi-square. The significance level was set at $0.01 \%$. The study was approved by the Research Ethics Committee. Results: For the 25 mothers in the study, basic sanitation was the only predictor of accuracy and totality in the KIDI. Percentage scores and standard deviation had correct answers on the inventory, with $0.90( \pm 0.07)$ in the attempt, $0.57( \pm 0.07)$ in accuracy, and $0.51( \pm 0.07)$ in the total score. Also, $0.34( \pm 0.08)$ of mothers underestimated the development and 0.14 $( \pm 0.06)$ overestimated it. Conclusion: Mothers' knowledge about child development in rural areas is related to sociodemographic factors. Basic sanitation was the only environmental predictor variable of accuracy and total correctness of the instrument, indicating to predict greater maternal knowledge when present.
\end{abstract}

Keywords: Health knowledge, Attitudes, practice, Child development, Cultural characteristics, Rural areas.

\footnotetext{
1Universidade Federal do Pará (UFPA), Belém - PA. *E-mail: ivetecaldas@uepa.br

2Universidade do Estado do Pará (UEPA), Marabá - PA.

3University of Glasgow, Scotland - UK.
} 


\section{RESUMEN}

Objetivo: Investigar factores ambientales y sociodemográficos relacionados con el conocimiento materno sobre desarrollo infantil en el contexto rural. Métodos: Estudio transversal, de madres con hijos entre 0 y 3 años, en condado de Pará. Se utilizó un formulario de identificación, caracterización ambiental y sociodemográfica y el Inventario de Conocimientos del Desarrollo Infantil. Para el análisis estadístico se utilizaron modelos lineales; para comparaciones múltiples, Sidak, y para las características de la muestra, la prueba de Chi-cuadrado. El nivel de significancia fue del 0,01\%. El estudio fue aprobado por el Comité de Ética en Investigación. Resultados: Para las 25 madres en el estudio, saneamiento básico fue el único con significación estadística. Para porcentuales y la desviación estándar, hubo respuestas correctas en las preguntas del inventario con $0.90( \pm 0.07)$ en el intento, $0.57( \pm 0.07)$ en precisión y $0.51( \pm 0.07)$ en el total. $0,34( \pm 0,08)$ de las madres subestimaron el desarrollo de sus hijos y $0,14( \pm 0,06)$ sobreestimaron. Conclusión: El conocimiento de las madres de áreas rurales sobre el desarrollo infantil está relacionado con factores sociodemográficos. Entre las variables ambientales saneamiento básico fue el único predictor de la exactitud y total corrección, lo que indica mayor conocimiento materno cuando presente.

Palabras clave: Conocimientos, Actitudes y práctica en salud desarollo infantil, Características culturales, Medio rural.

\section{INTRODUÇÃO}

O desenvolvimento infantil é caracterizado pela capacidade contínua da criança em se adaptar ao ambiente em que está inserida. É marcado por alterações físicas, cognitivas, comportamentais, neurológicas e sociais, que se expressam de forma gradual à medida que esta atinge a competência de corresponder às suas necessidades e às do ambiente (CALDAS IFR, et al., 2016).

Outros fatores que podem interferir na relação mãe-filho são as condições sociais, econômicas, demográficas e cognitivas. Quando essas são precárias, podem causar isolamento social e reduzir a eficácia do desenvolvimento. Ainda, estudos indicam que fatores sociodemográficos maternos, como nível socioeconômico, escolaridade, idade e número de filhos podem ser preditores significativos da compreensão sobre o desenvolvimento infantil (SCARZELLO D, et al., 2016; BORNSTEIN MH, et al., 2010).

A renda familiar e a escolaridade materna baixa podem acarretar elevado estresse familiar e padrões negativos no comportamento materno durante as interações com seu filho (CALDAS IFR, et al., 2016). Em contextos ambientais desfavoráveis, como moradias precárias, as mães podem apresentar dificuldade em reconhecer e responder aos sinais da criança, maior negatividade verbal, baixo calor afetivo, menor envolvimento e menos suporte às demandas da criança (CAVALCANTE MCB, et al., 2017).

Baseado nisso, populações rurais merecem destaque, pois vivem predominantemente em condições de vulnerabilidade, pobreza e baixa escolaridade. Geralmente são caracterizadas por populações com educação deficiente, oportunidades escassas de emprego, residências precárias, baixa renda familiar e dificuldade de acesso a água encanada e saneamento básico. Ademais, há escassez de transporte público, falta de serviços de saúde e de infraestrutura, como energia elétrica e meios de comunicação, tornando as condições de saúde dessa região mais precárias do que as da zona urbana (GONÇALVES H, et al., 2018).

Considerando a importância do contexto para a compreensão sobre o desenvolvimento infantil, pesquisadores apontam que mães da zona rural valorizam mais práticas de criação relacionadas à disciplina dos filhos, enquanto que mães urbanas com elevada escolaridade valorizam mais práticas de estimulação à criança; sendo assim, mães rurais tendem a ter maior prática em cuidados primários, enquanto mães de capitais apresentam maior prática na realização e valorização de cuidados relativos à estimulação neuropsicomotora (BORGES LC e SALOMÃO NMR, 2018).

Portanto, a compreensão materna sobre o desenvolvimento infantil representa grande potencial em favorecer a corresponsabilidade e autonomia no cuidado de seus filhos. Este estudo objetiva investigar os fatores ambientais e sociodemográficos que estão relacionados ao conhecimento materno sobre 0 desenvolvimento infantil no contexto rural. 


\section{MÉTODOS}

Trata-se de um estudo transversal com amostra por conveniência. A pesquisa foi realizada em vilas de município localizado no estado do Pará. O período da coleta de dados ocorreu entre agosto e novembro de 2018. Participaram 25 mães, cujos filhos tinham entre 0 e 3 anos de idade. Os critérios de inclusão foram mães biológicas de crianças entre 0 e 3 anos de idade, que recebem atendimento em uma Unidade de Saúde da Família (USF) no município. Foram excluídas mães portadoras de transtornos mentais, que tinham filhos com disfunções no desenvolvimento e aquelas que não participaram da entrevista completa.

A ficha de identificação e caracterização ambiental e sociodemográfica utilizada era composta por perguntas que acessavam informações sobre variáveis ambientais (tipo de moradia, acesso à água encanada, saneamento básico e renda familiar) e sociodemográficas relacionadas à mãe (faixa etária, escolaridade, número de gestações (incluindo abortos) e número de filhos).

A ferramenta Knowledge of Infant Development Inventory (KIDI) avaliava a proporção de conhecimento de pais relacionados a quatro domínios: normas e marcos do desenvolvimento; princípios; parentalidade; saúde e segurança (NOBRE-LIMA L, et al., 2014). O KIDI é composto por 58 perguntas de múltipla escolha, as quais na primeira fase - questões 1 a 39 (exemplos "uma criança de 2 anos que está 2 ou 3 meses atrasada de outras crianças de 2 anos está atrasada no seu desenvolvimento?", "bebês não podem ver e ouvir ao nascer?" e "conversar com uma criança sobre as coisas que ela está fazendo ajuda o desenvolvimento mental?") a mãe responde: "concordo", "discordo" ou "não tenho certeza", e na segunda fase - questões 40 a 58 (exemplos: "os bebês geralmente andam por volta dos 12 meses de idade?", "crianças de um ano sabem diferenciar o certo do errado?" e "os bebês costumam dizer sua primeira palavra real aos 6 meses?" as respostas são: "concordo", "mais jovem", "mais velho" e "não tenho certeza".

Esses modelos de respostas permite o cálculo de três pontuações percentuais: tentativa, precisão e total. A tentativa representa a frequência com que as mães não escolhem a opção "Não tenho certeza" e estão relacionados à confiança do conhecimento das mesmas. A precisão é a proporção de respostas corretas a partir de todas as respostas tentadas. E a pontuação total é o produto da tentativa e precisão, e reflete a porcentagem de respostas corretas de todos os itens no KIDI. Pontuações percentuais $\geq 70 \%$ na pontuação total do KIDI indica conhecimento adequado sobre o desenvolvimento infantil (MCMILLIN C, et al., 2016). As pontuações percentuais do KIDI foram apresentadas em números decimais.

As questões 40 a 58 também apresentam opções de resposta que permitem analisar a extensão do conhecimento impreciso, podendo mostrar se a mãe está subestimando ou superestimando as habilidades que a criança é capaz de realizar. Os cálculos de subestimação e superestimação também expõem pontuações percentuais, e são inversamente proporcionais ao nível de conhecimento sobre 0 desenvolvimento infantil (NOBRE-LIMA L, et al., 2014).

As mães foram abordadas na sala de espera, no dia da consulta agendada na USF que realiza a cobertura assistencial de todas as mães residentes em vilas do município. No momento do acolhimento, o pesquisador realizou esclarecimentos sobre a importância da pesquisa, os objetivos, além da não identificação pessoal nos instrumentos. Mediante o aceite voluntário em participar do estudo, concedido pela assinatura de Termo de Consentimento Livre e Esclarecido (TCLE), as mães foram encaminhadas para uma sala reservada nas dependências da USF para melhor comodidade e privacidade durante as entrevistas. A sala era arejada, com boa luminosidade, medindo $2 \times 3 \mathrm{~m}$ e possuía três cadeiras e uma mesa.

Cada entrevista durou 30 minutos, dividida em duas etapas em um único encontro. Na primeira etapa eram realizados 0 acolhimento da mãe e o registro na Ficha de identificação e caracterização ambiental e sociodemográfica (10 minutos), e na segunda, solicitava-se que a mãe respondesse as perguntas do KIDI (20 minutos). A coleta de dados foi conduzida em forma de entrevista individualizada pelo pesquisador. Este, lia em voz compassada e em bom tom cada instrumento, e as perguntas relacionadas, a mãe respondia oralmente. As respostas eram, então, registradas pelo pesquisador nos impressos dos instrumentos. Para análise estatística, foram utilizados modelos lineares generalizados, os quais são indicados para casos em que os pressupostos de normalidade não são atendidos. Dessa forma, os modelos lineares generalizados apenas requerem que a variável dependente esteja linearmente relacionada aos preditores. O teste Sidak foi usado para comparações múltiplas. Os dados ambientais e sociodemográficos 
relacionados à mãe foram descritos por frequências e porcentagens. As características da amostra foram comparadas pelo Teste de Qui-Quadrado (x2). O nível de significância foi estabelecido em 0,01. A presente pesquisa foi aprovada pelo Comitê de Ética e Pesquisa com seres humanos da Universidade Federal do Pará (UFPA) expressa no parecer ( $n^{\circ}$ 2.892.917) respeitada a Resolução do Ministério da Saúde CNS/MS no 466/12, com a participação condicionada à assinatura do Termo de Consentimento Livre e Esclarecido (TCLE) pelas entrevistadas.

\section{RESULTADOS}

Referente às características ambientais e sociodemográficas relacionadas às mães participantes do estudo, a maioria (52.0\%) reside em casa própria, $80.0 \%$ não possui saneamento básico, $88.0 \%$ apresenta renda familiar menor que um salário mínimo, 56.0\% tem entre 20 e 29 anos de idade, $60.0 \%$ possui ensino fundamental incompleto e $40.0 \%$ tem apenas um filho (Tabela 1).

Tabela 1 - Características ambientais e sociodemográficas relacionadas às mães participantes do estudo, $\mathrm{n}=25$.

\begin{tabular}{|c|c|c|c|}
\hline \multirow{2}{*}{ Variáveis } & \multicolumn{3}{|c|}{ Amostra Total $(n=25)$} \\
\hline & $\mathbf{n}$ & $\%$ & p valor ${ }^{(1)}$ \\
\hline \multicolumn{4}{|c|}{ Características Ambientais } \\
\hline \multicolumn{4}{|l|}{ Tipo de moradia } \\
\hline Própria & 13 & 52.0 & \multirow{3}{*}{0.733} \\
\hline Alugada & 0 & 0.0 & \\
\hline Favor & 12 & 48.0 & \\
\hline \multicolumn{4}{|l|}{ Água encanada } \\
\hline Sim & 18 & 72.0 & 0.797 \\
\hline \multicolumn{4}{|l|}{ Saneamento básico } \\
\hline Sim & 5 & 20.0 & \multirow{2}{*}{$0.001^{* *}$} \\
\hline Não & 20 & 80.0 & \\
\hline \multicolumn{4}{|l|}{ Renda familiar } \\
\hline < 1 salário mínimo & 22 & 88.0 & \multirow{3}{*}{0.623} \\
\hline 1 salário mínimo & 3 & 12.0 & \\
\hline > 1 salário mínimo e < 2 & 0 & 0.0 & \\
\hline \multicolumn{4}{|c|}{ Características Sociodemográficas } \\
\hline \multicolumn{4}{|l|}{ Faixa etária (anos) } \\
\hline $14-19$ & 7 & 28.0 & \multirow{3}{*}{0.941} \\
\hline $20-29$ & 14 & 56.0 & \\
\hline $30-39$ & 4 & 16.0 & \\
\hline \multicolumn{4}{|l|}{ Escolaridade } \\
\hline Ensino fundamental incompleto & 15 & 60.0 & \multirow{6}{*}{0.769} \\
\hline Ensino fundamental completo & 2 & 8.0 & \\
\hline Ensino médio incompleto & 5 & 20.0 & \\
\hline Ensino médio completo & 3 & 12.0 & \\
\hline Ensino superior incompleto & 0 & 0.0 & \\
\hline Ensino superior completo & 0 & 0.0 & \\
\hline \multicolumn{4}{|l|}{ № de gestações } \\
\hline 1 & 10 & 40.0 & \multirow{3}{*}{0.561} \\
\hline 2 & 7 & 28.0 & \\
\hline 3 ou mais & 8 & 32.0 & \\
\hline \multicolumn{4}{|l|}{$\mathbf{N}^{\circ}$ de filhos } \\
\hline 1 & 11 & 44.0 & \multirow{3}{*}{0.638} \\
\hline 2 & 7 & 28.0 & \\
\hline 3 ou mais & 7 & 28.0 & \\
\hline
\end{tabular}

Legenda: ${ }^{(1)}$ Teste Qui-Quadrado ( $p$ valor $\leq 0.01$ ).

Fonte: Sousa JR, et al., 2020.

Em relação às pontuações percentuais e desvio padrão do KIDI, os resultados do estudo mostram acertos sobre as perguntas do inventário com $0.90( \pm 0.07)$ na tentativa, $0.57( \pm 0.07)$ na precisão e 0.51 
$( \pm 0.07)$ na pontuação total. $E$ quanto as pontuações percentuais de subestimação (under) $e$ superestimação (over), $0.34( \pm 0.08)$ das mães subestimam o desenvolvimento de seus filhos e 0.14 ( \pm 0.06$)$ superestimam. Os modelos lineares generalizados revelam que em relação às variáveis ambientais, 0 saneamento básico foi o único preditor de precisão e total do KIDI. As mães que habitam em moradias com saneamento básico apresentam maior porcentagem de precisão $(0.62 \pm 0.02)$ quando comparadas àquelas que vivem em moradias sem tais condições $(0.55 \pm 0.02)(x 2=4.123, d f=1, p=0.042)$. Resultados semelhantes foram encontrados para pontuação total do KIDI, em que aquelas que possuem saneamento básico também apresentam maior porcentagem $(0.57 \pm 0.01)$, diferente daquelas que não possuem $(0.49 \pm 0.01)(X 2=11.592$, $d f=1, p=0.001)$.

Quanto às variáveis sociodemográficas relacionadas à mãe, faixa etária, escolaridade, número de gestações e número de filhos foram preditores de precisão, tentativa e total do KIDI. Em relação a pontuação percentual de tentativa, mães com ensino fundamental completo apresentam maior porcentagem $(0.99 \pm 0.02)$ do que aquelas com ensino médio incompleto $(0.89 \pm 0.02) \quad(x 2=16.488, d f=3, p=0.001)$. Ademais, mães com única gestação apresentam maior porcentagem $(1.07 \pm 0.03)$ quando comparadas com mães com duas gestações $(0.89 \pm 0.03)$ e com três ou mais $(0.85 \pm 0.03) \quad(x 2=10.481, d f=1, p=0.001)$. $E$ aquelas com dois filhos destacam-se por apresentarem maior porcentagem $(1.00 \pm 0.02)$ diferente daquelas com três ou mais $(0.99 \pm 0.034)$ e um único filho $(0.82 \pm 0.02)\left(x^{2}=16.898, d f=2, p=0.0001\right)$ (Figura 1).

Figura 1 - Pontuação percentual de tentativa do knowledge of infant development inventory (KIDI), quanto a escolaridade, número de gestações e número de filhos.

A

B
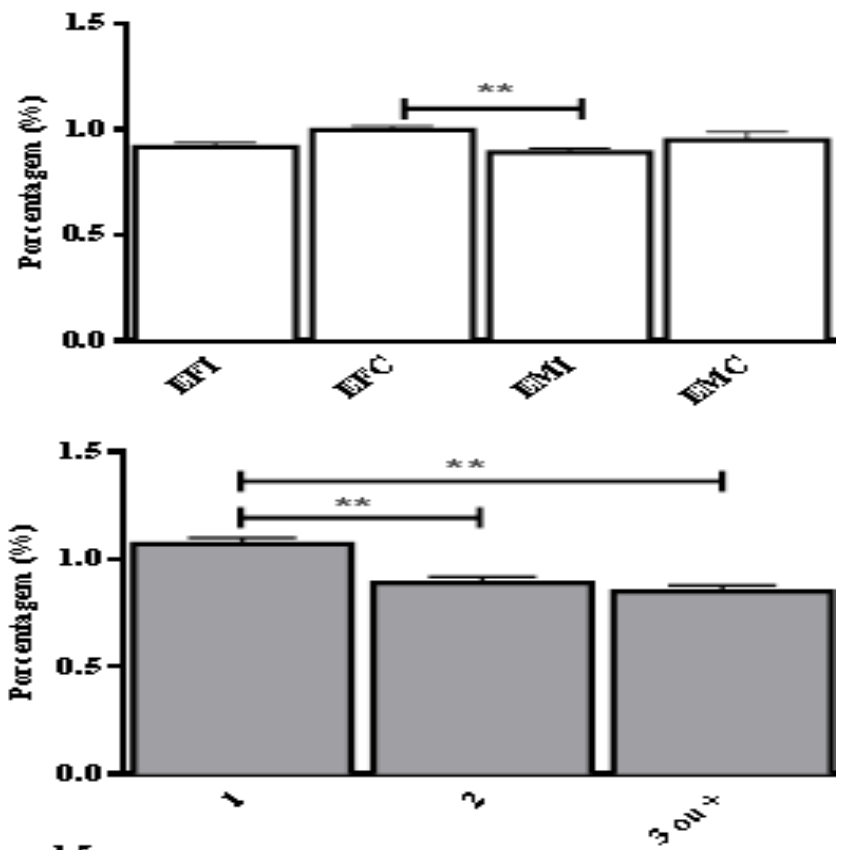

C

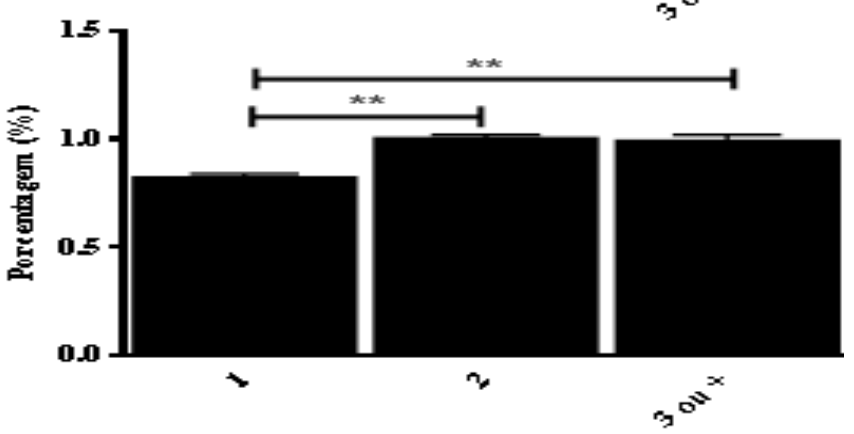

Legenda: (A) escolaridade; (B) número de gestações; (C) número de filhos; EFI (ensino fundamental incompleto); EFC (ensino fundamental completo); EMI (ensino médio incompleto); EMC (ensino médio completo). Teste de Sidak ( $\left.{ }^{* *} p<0.0001,{ }^{*} p<0.001\right)$. Fonte: Sousa JR, et al., 2020. 
As variáveis faixa etária e número de filhos também foram preditores de precisão do KIDI. Mães entre 20-29 anos apresentam maior porcentagem (0.57 \pm 0.02$)$ quando comparadas com mães entre 30-39 anos $(0.49 \pm 0.02)(x 2=8.489, d f=1, p=0.014)$, e aquelas com apenas um filho apresentam maior porcentagem $(0.61 \pm 0.01)$ quando comparadas às mães com três ou mais $(0.58 \pm 0.02)$ e dois filhos $(0.45 \pm 0.01)$ $(x 2=81.093, d f=2, p=0.000)$ (Figura 2).

Figura 2 - Pontuação percentual de precisão do Knowledge of Infant Development Inventory (KIDI) quanto a faixa etária e número de filhos.

A

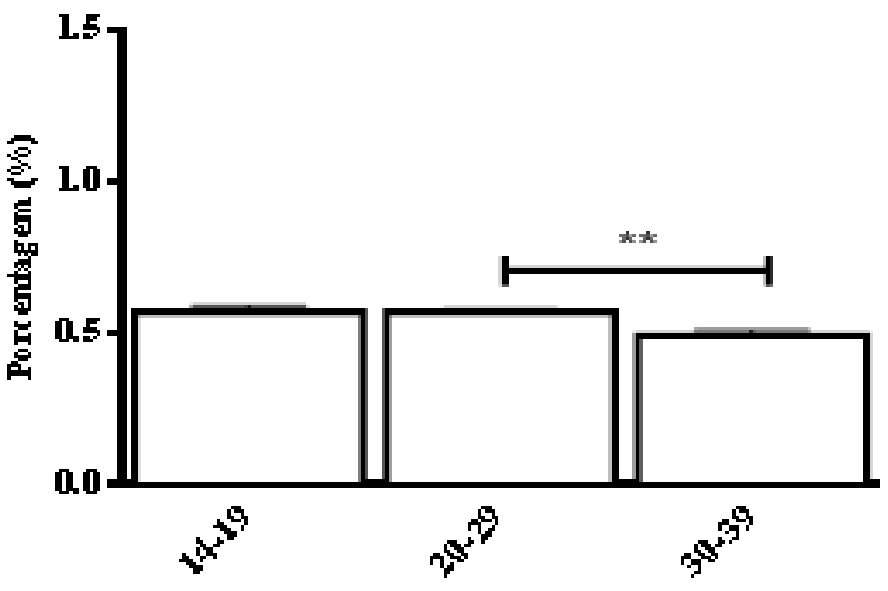

B

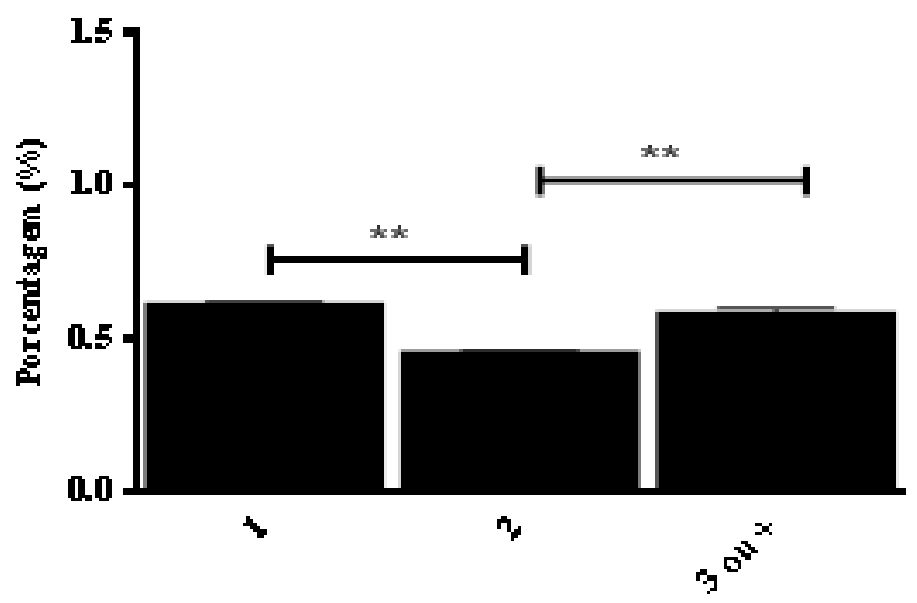

Legenda: (A) faixa etária; (B) número de filhos. Teste de Sidak $\left({ }^{* *} p<0.0001,{ }^{*} p<0.001\right)$.

Fonte: Sousa JR, et al., 2020.

Por fim, o número de filhos também foi preditor de subestimação (under) no KIDI. Mães com um filho apresentam maior porcentagem $(0.61 \pm 0.01)$ quando comparadas àquelas com dois $(0.45 \pm 0.01)(X 2=6.8753$, $\mathrm{df}=2, \mathrm{p}=0.032$ ). Já em relação a superestimação (over), as variáveis faixa etária, número de gestações e número de filhos foram preditores. Mães entre 30-39 anos apresentam maior porcentagem (0.22 \pm 0.03$)$ quando comparadas com mães entre $20-29$ anos $(0.13 \pm 0.01)(x 2=6.143, d f=2, p=0.046)$.

Aquelas com única gestação $(0.30 \pm 0.05)$ apresentam maior porcentagem $(0.30 \pm 0.05)$ diferente daquelas com 3 ou mais gestações $(0.04 \pm 0.04) \quad(x 2=8.366, d f=2, p=0.015)$. $E$ as mães com 3 ou mais filhos $(0.25 \pm 0.05)$ apresentam maior pontuação quando comparadas aquelas com dois filhos $(0.24 \pm 0.03)$ e com um filho $(0.02 \pm 0.04)(0.25 \pm 0.05)(x 2=9.671, \mathrm{df}=2, \mathrm{p}=0.008)$ (Figura 3). 
Figura 3 - Pontuação percentual de superestimação (Over) do Knowledge of Infant Development Inventory (KIDI) referente à faixa etária, número de gestações e número de filhos.

A

B
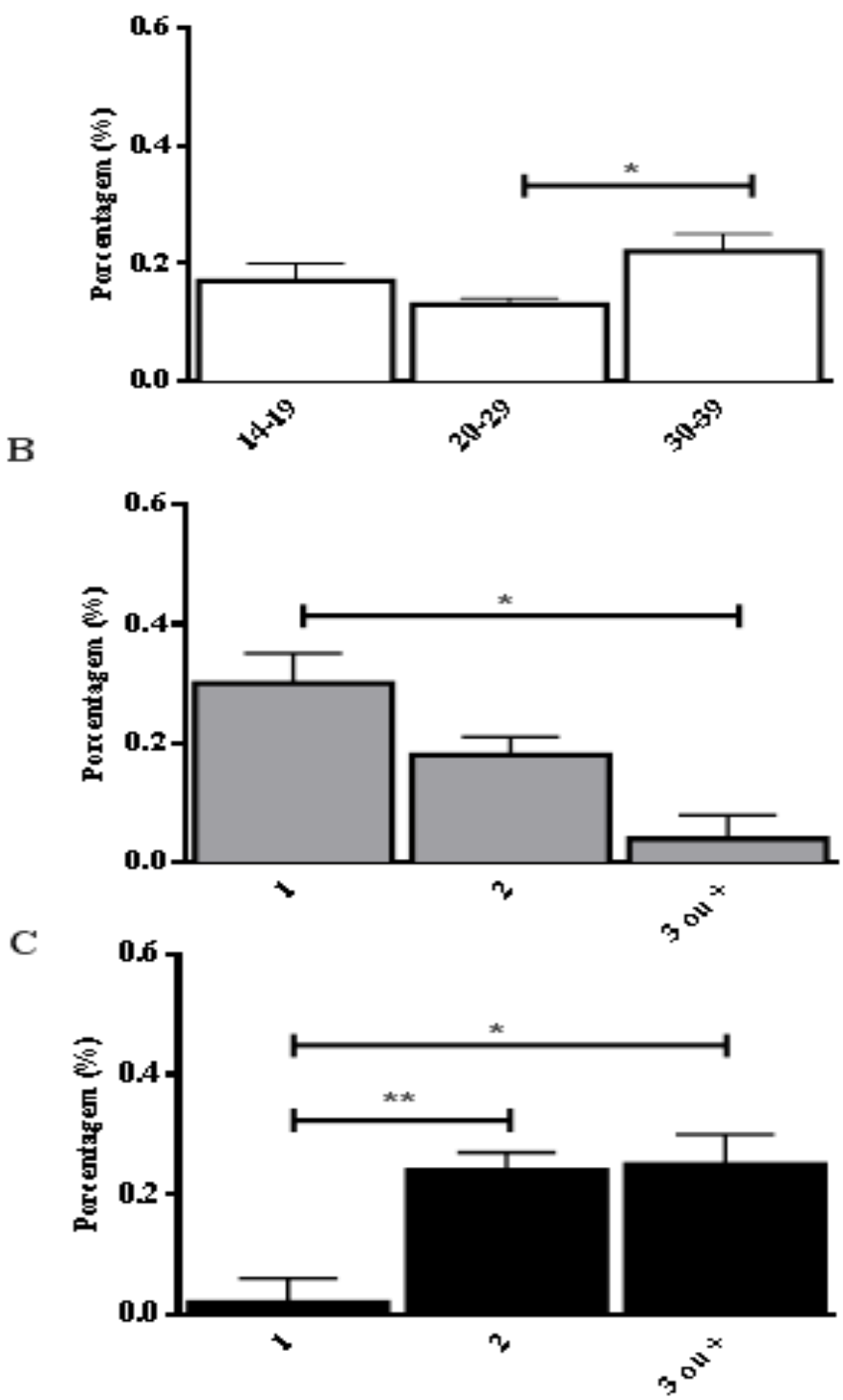

Legenda: (A) faixa etária; (B) número de gestações; (C) número de filhos. Teste de Sidak $\left({ }^{* *} \mathrm{p}<0.0001\right.$, $\left.{ }^{*} \mathrm{p}<0.001\right)$.

Fonte: Sousa JR, et al., 2020.

\section{DISCUSSÃO}

O conhecimento materno sobre o desenvolvimento infantil, processo no qual ocorrem mudanças físicas, cognitivas e comportamentais, tem fundamental importância pois influencia as crenças e práticas parentais e, consequentemente o desenvolvimento dos seus filhos. Estudo aponta que mães que têm melhor conhecimento tendem a oferecer mais oportunidades para o desenvolvimento social e cognitivo dos filhos (AL-MAADADI F e IKHLEF AE, 2015; NUTALL AK, et al., 2015).

Nesse sentido, em relação ao conhecimento materno sobre o desenvolvimento infantil, pontuações percentuais $\leq 70 \%$ no KIDI são potencialmente indicativas de déficits de conhecimento, com níveis significativamente mais baixos de empatia da mãe (MCMILLIN SE, et al., 2016).

MacPhee D (2002), autor do instrumento utilizado neste estudo, ao avaliar mães norte-americanas constatou pontuação de $0.72( \pm 0,12)$, enquanto em nosso estudo as mães da zona rural apresentaram 0.51 
$( \pm 0,07)$, indicando baixo conhecimento. Todavia, uma pesquisa realizada no Egito sobre a percepção de mães rurais e urbanas sobre o desenvolvimento de crianças menores de dois anos mostrou que em ambos os contextos, não houve boa compreensão das etapas das habilidades motoras, cognitivas e sociais dos seus filhos (ELGIBALY O e AZIZ MM, 2016).

Além disso, encontrou-se que o saneamento básico, dentre as variáveis ambientais é o único preditor da precisão e do total de acertos no instrumento KIDI, em que a presença dessas condições nas moradias da zona rural parece predizer maior conhecimento materno. Semelhantemente à presente pesquisa, mães de área periférica no interior do Estado de São Paulo que vivem em condições socioeconômicas precárias também apresentaram baixo conhecimento sobre o desenvolvimento de seus filhos (MATSUKURA TS, et al., 2014).

A evolução do desenvolvimento infantil pode ser influenciada por fatores como hereditariedade, nível socioeconômico, cultura, etnia e nível de conhecimento dos pais sobre esse processo (PRADO CMCS e CAMPOMORI MVCC, 2019; MACPHEE D, 2002). Nesse sentido, quanto às características sociodemográficas relacionadas à mãe no presente estudo, apenas as variáveis escolaridade, número de gestações e número de filhos foram preditores de tentativa do KIDI.

Utilizando o mesmo instrumento de avaliação do conhecimento materno, um estudo também observou que o nível de escolaridade materna e ter mais de um filho influenciam positivamente no conhecimento sobre o desenvolvimento infantil. Entretanto, a confiança materna pode ser influenciada tanto pelo nível de escolaridade, como por experiências vivenciadas por ela (BORNSTEIN MH, et al., 2010).

Encontrou-se, nesta pesquisa, que as mães com ensino fundamental completo tiveram maior confiança no próprio conhecimento do que aquelas com ensino médio incompleto, esse resultado nos possibilita a questionar se a confiança das mães poderia estar mais ligada ao conhecimento "informal" adquirido das experiências parentais oriundas de gerações anteriores do que ao conhecimento "formal" oferecido por meio da escola, por exemplo. O número de gestações, por sua vez, foi preditor negativo de tentativa. As mães com gestação única apresentaram maior porcentagem quando comparadas àquelas com duas e três ou mais gestações. Hipotetiza-se que a primeira gestação, por ser geralmente a mais desejada, possa conferir maior dedicação e sentimento de amor incondicional da mãe ao filho, portanto, maior envolvimento materno em atividades relacionadas aos cuidados infantis (AL-MAADADI F e IKHLEF AE, 2015).

Por outro lado, um estudo identificou que a confiança das mães foi maior em genitoras com mais filhos, podendo estar relacionada à experiência direta vivenciada nos cuidados dos demais filhos (MACKINNON K, et al., 2015). Ainda, foi evidenciado no estudo que mães mais velhas apresentam pontuação menor de precisão no $\mathrm{KIDI}$, diferente do estudo que mostrou haver maior conhecimento sobre o desenvolvimento infantil com anos de experiência de vida (BORGES LC e SALOMÃO NMR, 2018). Ademais, mães mais jovens podem estar mais propensas ao acesso a informações do que mães mais velhas, apesar da zona rural ter acesso mais restrito (ESCOSTEGUY ACD e FELIPPI ACT, 2017).

Identificou-se também que mães com dois filhos tiveram maior pontuação de precisão quando comparadas a mães com um ou 3 ou mais filhos. Acredita-se que genitoras com apenas um filho podem ter menor experiência em cuidados infantis que aquelas com mais filhos. Ademais, as mudanças vivenciadas pela mãe em sua primeira gestação irão influenciar sua adaptação em cuidar do primeiro filho, como exemplo, ganhar o papel de mãe e perder o papel de filha, refletindo em novas responsabilidades e alterações emocionais (MACKINNON K, et al., 2015).

Por outro lado, a literatura mostra que a taxa de pobreza em famílias com 3 ou mais filhos é maior em comparação a famílias com um ou dois filhos, uma vez que ocorre participação limitada do pai no cuidado, pois necessita trabalhar fora do domicílio. Consequentemente, a mãe torna-se a principal responsável na criação dos filhos, podendo sentir-se sobrecarregada e menos atenta às questões relacionadas ao desenvolvimento (SALINAS RC, et al., 2017).

Outro ponto que merece destaque são os resultados da extensão do conhecimento impreciso, que analisou se a mãe superestima ou subestima as habilidades que a criança é capaz de realizar. Estimativas 
excessivas (superestimação) sobre o desenvolvimento infantil podem levar à frustração por parte da criança e da mãe, aumentando a punição sobre a criança, enquanto que subestimações, ou seja, esperar dela menos do que é capaz, pode levar a estimulação menos apropriada para o desenvolvimento (AL-MAADADI F e IKHLEF AE, 2015).

As percepções são influenciadas pela relação entre a pessoa que percebe e a pessoa que é percebida, bem como pelas experiências, convicções e características do percebedor que afetarão pensamentos, sentimentos e atitudes com relação à pessoa percebida (WARKENTIN S, et al., 2018). Nesse sentido, encontrou-se no presente estudo que mães mais velhas superestimaram mais o desenvolvimento do que aquelas mais novas, de modo que essas podem ter adquirido mais experiências observando ou cuidando de muitas crianças (de amigos, familiares ou vizinhos).

Por outro lado, mães com 3 ou mais gestações superestimaram menos o desenvolvimento dos seus filhos do que àquelas com número inferior de gestações. A Organização Mundial de Saúde (OMS) preconiza a realização de no mínimo sete consultas pré-natais, quando a gestante recebe atenção específica e olhar atento no desenvolvimento da gravidez, assim como após o parto (VALE CLQ, et al., 2017).

Com isso, o maior número de gestações pode ter favorecido o contato da mãe com a equipe de saúde e a construção de maior vínculo e conhecimentos sobre os cuidados de saúde, solucionando dúvidas sobre desenvolvimento dos seus filhos. As mães primigestas também superestimaram menos o desenvolvimento dos seus filhos. Acredita-se que na presença de um único filho a atenção materna é exclusiva, fortalecendo o vínculo afetivo e sociocomunicativo. Essas, portanto, são mais atentas ao desenvolvimento e às necessidades do seu filho, sendo mais precisas quanto às habilidades que o mesmo é capaz de realizar em cada etapa da vida (ZANETTINI A, et al., 2019). O fato de a presente pesquisa não ter investigado sobre as experiências maternas sobre o cuidado parental constitui uma limitação do estudo.

\section{CONCLUSÃO}

Os resultados do presente estudo sugerem que o conhecimento das mães sobre o desenvolvimento infantil no contexto rural difere conforme idade, o nível socioeconómico e o número de filhos. Dentre as variáveis ambientais identificadas no presente estudo, apenas o saneamento básico foi preditor de conhecimento materno sobre o desenvolvimento infantil, enquanto dentre as variáveis sociodemográficas relacionadas à mãe, a faixa etária, o nível de escolaridade, o número de gestações e o número de filhos foram preditores, reforçando que características sociodemográficas estão mais fortemente relacionadas ao conhecimento de mães da zona rural. Dessa forma, essa pesquisa contribui de forma importante para a visualização da realidade a respeito dos fatores ambientais e sociodemográficos que estão relacionados ao conhecimento materno sobre o desenvolvimento infantil no contexto rural. Reitera-se a relevância de pesquisas que priorizem análises com maiores amostras e contextos culturais diferentes, com o intuito de identificar outras diferenças.

\section{REFERÊNCIAS}

1. AL-MAADADI F, IKHLEF AE. What Mothers Know About Child Development and Parenting in Qatar: Parenting Cognitions and Practices. The Family Journal, 2015; 23(1): 65-73.

2. BORGES LC, SALOMÃO NMR. Práticas de educação dos filhos em contexto não urbano. Psicologia em Revista, 2018; 24(1): 175-190.

3. BORNSTEIN MH, et al. Parenting knowledge: experiential and sociodemographic factors in European American mothers of young children. Developmental Psychology, 2010; 46(6): 1677-1693.

4. CALDAS IFR, et al. Fatores de risco e desenvolvimento sociocomunicativo em prematuros. Revista PsicologiaTeoria e Prática, 2016; 18(2): 129-141.

5. CAVALCANTE MCB, et al. Relação mãe-filho e fatores associados: análise hierarquizada de base populacional em uma capital do Brasil-Estudo BRISA. Ciência \& Saúde Coletiva, 2017; 22(5): 1683-1693.

6. ELGIBALY O, AZIZ MM. Assessment of the needs of mothers and primary healthcare providers to support early childhood development in Egypt: a qualitative study. Child: Care, Health and Development, 2016; 42(3), 394-401.

7. ESCOSTEGUY ACD, FELIPPI ACT. Ruralidade e tecnologias de informação e comunicação: os novos modos de viver de famílias agricultoras. Cuadernos del Claeh, 2017; 36(106): 125-144.

8. GONÇALVES $\mathrm{H}$, et al. Estudo de base populacional na zona rural: metodologia e desafios. Revista Saúde de Publica, 2018, 52 Supl 1:3s. 
9. MACPHEE D. Knowledge of Infant Development Inventory Manual. Colorado State University. Forthcoming, 2002.

10. MATSUKURA TS, et al. Saúde mental infantil em contextos de desvantagem socioeconômica: fatores de risco e proteção. Cadernos Brasileiros de Terapia Ocupacional, 2014; 22(2): 251-262.

11. MACKINNON K, et al. Student and educator experiences of maternal-child simulation-based learning: a systematic review of qualitative evidence protocol. JBI Database of Systematic Reviews \& Implementation Reports, 2019; 13(1): 14-26.

12. MCMILLIN SE, et al. The Role of Maternal Knowledge of Child Development in Predicting Risk for Child Maltreatment. Clinical Pediatrics, 2016; 55(4): 374-6.

13. NOBRE-LIMA L, et al. The Portuguese version of the Knowledge of Infant Development Inventory-P (KIDIP). European Journal of Developmental Psychology, 2014; 11(6): 740-45.

14. NUTTALL AK, et al. Maternal History of Parentification and Warm Responsiveness: The Mediating Role of Knowledge of Infant Development. Journal of Family Psychology, 2015; 29(6): 863-72.

15. PRADO CMCS, CAMPOMORI MVCC. Desenvolvimento neuromotor infantil: produção técnico-cultural visual para complementar a educação permanente do público-alvo na atenção primária de saúde. Revista Interdisciplinar de Extensão, 2019; 3(5): 22-40.

16. SALINAS RC, et al. Impacto de composición familiar en los niveles de pobreza de Perú. Cultura-hombre-sociedad, 2017; 27(2): 69-88.

17. SCARZELLO D, et al. Parental practices of Italian mothers and fathers during early infancy: The role of knowledge about parenting and child development. Infant Behavior and Development, 2016; 44:133-43.

18. VAN DER VOORT A, et al. Sensitive parenting is the foundation for secure attachment relationships and positive social-emotional development of children. Journal of Children's Services, 2014; 9(2): 165-176.

19. VALE CLQ, et al. Percepção de gestantes sobre o pré-natal. Revista Interdisciplinar, 2017; 10(4): 39-49.

20. WARKENTIN S, et al. Factors associated with parental underestimation of child's weight status. Jornal de Pediatria, 2018; 94(2): 162-169.

21. ZANETTINI A, et al. As Vivências da Maternidade e a Concepção da Interação Mãe-Bebê: Interfaces Entre as Mães Primíparas Adultas e Adolescentes. Journal of Research: Fundamental Care Online, 2017; 11(3): 655-663. 